\title{
Оценка эффективности
}

\section{конструкторско-технологических решений по снижению виброактивности приводных зубчатых механизмов на основе анализа собственных форм колебаний}

\author{
Н.Н. Ишин, А.М. Гоман, А.С. Скороходов \\ Государственное научное учреждение «Объединенный институт машиностроения» Национальной \\ академии наук Беларуси, 220072, Минск, Республика Беларусь, Академическая ул., д. 12
}

\section{The Evaluation of the Effectiveness of Design and Technological Solutions to Reduce Vibration Activity of Drive Gear Mechanisms Based on the Analysis of Normal Vibration Modes}

\author{
N.N. Ishin, A.M. Goman, A.S. Skorokhodov \\ The State Scientific Institution - Joint Institute of Mechanical Engineering of the National Academy of Sciences \\ of Belarus, 220072, Minsk, Republic of Belarus, Akademicheskaya St., Bldg 12 \\ e-mail: nik_ishin@mail.ru, ark.goman@gmail.com, skanst@yandex.ru
}

В настоящее время практически перед всеми производителями техники достаточно остро стоит задача улучшения виброакустических характеристик приводных зубчатых механизмов. В связи с этим важно уже на стадии проектирования оценить целесообразность применения новых конструкторско-технологических решений зубчатых колес и выбор их параметров с точки зрения оптимизации динамических качеств привода. Это можно осуществить на основе сравнительной оценки качества динамических систем по данным анализа их собственных форм колебаний. Целесообразность такого подхода объясняется тем, что изначально отсутствуют данные о диссипативных характеристиках сопряжений, деталей и материалов упругих элементов. Предложено при сопоставлении вариантов динамических схем использовать критерий виброактивности $H_{k}$, в выражение которого дополнительно введен коэффициент, характеризующий отношение параметров ударных импульсов, возникающих в зацеплении сопоставляемых систем. При этом форма возбуждающих импульсов в зубчатом зацеплении приводных зубчатых механизмов принята в виде полуволны синусоиды, отличающейся от общепризнанной в настоящее время прямоугольной формы. Рассмотрен пример, в котором критерий $H_{k}$ применен для оценки эффективности конструктивных решений по снижению виброактивности зубчатой передачи путем использования составного зубчатого колеса с упругим креплением и повышенной податливостью зубчатого венца. В результате уточненных расчетов получено значение критерия виброактивности сопоставляемых динамических схем, которое лучше согласуется с результатами экспериментальных исследований.

Ключевые слова: зубчатая передача, собственные частоты, собственные формы колебаний, ударный импульс, критерий виброактивности, составное зубчатое колесо. 
At present, practically all machine manufacturers face the problem of improving vibroacoustic characteristics of drive gear mechanisms. In this regard, it is important at the design stage to assess the feasibility of new design and technological solutions and the choice of gear parameters from the point of view of optimization of the dynamic characteristics of the drive. It can be achieved through a comparative quality assessment of dynamic systems based on the analysis of their normal modes. The rationale for this approach lies in the fact that initially there is no consistent data about dissipative characteristics of junctions, parts and materials of the elastic elements. When comparing various dynamic schemes, it is proposed to use the vibration activity criterion Hk. It is expressed using an additionally introduced coefficient that characterizes the relation of the parameters of the shock pulses occurring when the compared systems are engaged. The shape of the excitation pulses in the meshing of the drive gears is adopted in the form of half-sine waves, which differs from the generally accepted rectangular shape. The authors consider an example in which the Hk criterion is applied for evaluating the efficiency of design solutions to reduce vibration activity of gears by using a compound gear with elastic fastening and high compliance of the toothing. As a result of the refined calculations, the value of the vibration activity criterion of the compared dynamic schemes is obtained. This value is better correlated with the results of experimental studies.

Keywords: gear, normal frequencies, normal modes, shock pulse, vibration activity criterion, compound gear.

Методы оценки выбора рациональных параметров приводных зубчатых механизмов по критерию виброактивности зубчатых передач, основанные на анализе собственных форм колебаний динамических моделей сравниваемых вариантов конструкторских решений, достаточно глубоко рассмотрены в работах [1-3]. Данный подход также можно использовать при выборе рациональных физико-механических параметров покрытий зубьев (материал, толщина), применяемых для улучшения динамического качества зубчатых передач [4], при фланкировании и других конструкторско-технологических решениях.

При этом предполагается, что известна область $G$ рабочего диапазона частот возбуждающих колебаний. Для применения критерия определяют собственные частоты двух исследуемых динамических систем приводных зубчатых механизмов $\omega_{i}$ и $\omega_{j}$ (например, исходных серийно выпускаемых зубчатых колес и колес усовершенствованных с целью снижения виброактивности). Далее находят и сравнивают суммы значений виброускорений, определяемых по нормированным амплитудам собственных форм колебаний и собственным частотам из указанной области рабочего диапазона частот, по выражению [2]

$$
H_{k}=\frac{\sum_{\omega_{j} \in G}\left|\tilde{q}_{m_{k}}^{(j)}\right| \omega_{j}^{2}}{\sum_{\omega_{i} \in G}\left|\tilde{q}_{m k}^{(i)}\right| \omega_{i}^{2}},
$$

где $\tilde{q}_{m_{k}}^{(i)}$ и $\tilde{q}_{m_{k}}^{(j)}-$ нормированные амплитуды массы $m_{k}$, соответствующие собственным частотам $\omega_{i}$ и $\omega_{j}$ сопоставляемых систем.

Каждой собственной частоте $\omega_{j}$ соответствует определенная собственная $j$-я форма колебаний, т. е. определенные соотношения между амплитудными отклонениями масс системы $q_{m_{1}}^{(j)}, q_{m_{2}}^{(j)}, \ldots, q_{m_{k-1}}^{(j)}, q_{m_{k}}^{(j)}, \ldots, q_{m_{n}}^{j}$. Нормированные амплитуды собственных форм определяют из отношения $\tilde{q}_{m_{k}}^{(j)}=q_{m_{k}}^{(j)} / N^{(j)}$, где

$$
N^{(j)}=\sum_{k=1}^{n}\left|q_{m_{k}}^{(j)}\right|
$$

Критерий (1) позволяет проводить на стадии проектирования предварительный анализ и сравнение виброакустических свойств сложных механических систем без решения задач вынужденных колебаний, требующих учета демпфирования во всем спектре рабочих частот $[1$, 5]. Однако, как видно из выражения (1), такой метод не учитывает соотношения и взаимосвязи амплитуд внешних возмущающих воздействий на динамическую систему в исследуемом рабочем диапазоне частот.

Цель работы - развитие метода оценки уровня виброактивности сопоставляемых конструкционных решений зубчатых передач на стадии проектирования путем учета в критерии виброактивности параметров ударных импульсов, возникающих при пересопряжении зубьев. Влияние указанных возмущающих воздействий учитывается путем введения дополнительных коэффициентов, которые получают при расчете 
параметров ударных импульсов, возникающих в зубчатом зацеплении.

Критерий виброактивности зубчатых передач с учетом воздействия динамических ударных импульсов в зубчатом зацеплении. Динамическое воздействие на приводной зубчатый механизм можно представить в виде периодически повторяющихся ударных импульсов (далее под ударным импульсом будем понимать закон изменения ударной силы во времени) [2] с зубцовой частотой $f_{z}=n z / 60, \mathrm{c}^{-1}$ $\left(n-\right.$ частота вращения зубчатого колеса, мин $^{-1}$; $z$ - число зубьев). Эти импульсы обусловлены кромочными или срединными ударами в зубчатом зацеплении.

В работах $[2,6]$ форма ударного импульса динамического взаимодействия зубьев в первом приближении принята прямоугольной. Амплитуда такого импульса

$$
A_{r}=m \frac{v_{0}}{T}
$$

где $m-$ приведенная к основной окружности масса зацепляющихся колес; $v_{0}-$ скорость кромочного удара, сообщаемая колесу при контакте зубьев в нерасчетной точке; $T$ - длительность импульса.

При этом предполагается, что отношение длительности импульса $T$ к периоду зубцовой частоты $T_{z}$ не превосходит $T / T_{z}=0,1$.

Теоретические и экспериментальные исследования показали, что периодические ударные импульсы описываются полуволной синусоиды длительностью $T$ с максимальным (амплитудным) значением $F_{\max }$ [7]. Установлено, что в случае прямоугольной формы импульса максимальное значение ударной силы и время удара определяются со значительной погрешностью.

Амплитуды вынужденных колебаний элементов механизма на зубцовых и кратных им частотах в области собственных частот значи-

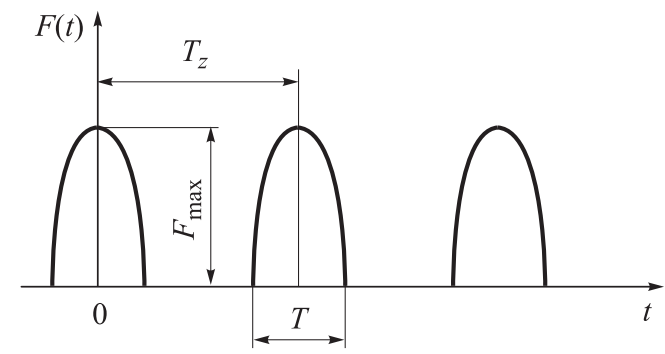

Рuc. 1. Периодические ударные импульсы тельно выше амплитуд на других частотах и взаимосвязаны между собой. Это обстоятельство учитывается при расчете суммы виброускорений зубчатого колеса механизма путем введения весовых коэффициентов $\beta_{i, j}$ при нормированных амплитудах собственных форм колебаний [2].

Эти коэффициенты находят посредством разложения в ряд Фурье действующих в зубчатой передаче ударных импульсов с периодом $T_{z}$ (рис. 1), определяемым по формуле

$$
T_{z}=\frac{1}{f_{z}}=\frac{60}{n z} .
$$

Для упрощения расчетов начало координат следует поместить в точку максимума импульса (см. рис. 1).

В этом случае форма ударного импульса выражается косинусоидальной функцией

$$
F(t)=F_{\max } \cos \frac{\pi}{T} t, \quad-\frac{T}{2} \leq t \leq \frac{T}{2} .
$$

Тогда ряд Фурье имеет вид

$$
F(t)=F_{0}+\sum_{k=1}^{\infty} F_{k} \cos \omega_{k} t
$$

где $\omega_{k}$ - гармоники, частоты которых кратны основной частоте $p_{z}=2 \pi / T_{z}, \omega_{k}=k p_{z}(k-$ номера гармоник, составляющие натуральный ряд чисел, $k=2,3,4, \ldots)$.

Коэффициент ряда Фурье $F_{k}$ определяется выражением

$$
\begin{gathered}
F_{k}=\frac{2}{T_{z}} \int_{-T_{z} / 2}^{T_{z} / 2} F_{\max } \cos \frac{\pi}{T} t \cos \omega_{k} t d t= \\
=\frac{4 T}{\pi T_{z}} F_{\max } \frac{\cos \frac{\omega_{k} T}{2}}{1-\left(\frac{\omega_{k} T}{\pi}\right)^{2}}=\frac{4 T}{\pi T_{z}} F_{\max } \frac{\cos \frac{k \pi T}{T_{z}}}{1-\left(\frac{2 k T}{T_{z}}\right)^{2}} .
\end{gathered}
$$

Весовые коэффициенты $\beta_{i, j}$ для нормированных амплитуд собственных форм колебаний находят из соотношения

$$
\beta_{i, j}=\frac{F_{k}}{F_{k=1}}=\frac{\left[1-\left(\frac{p_{z} T}{\pi}\right)^{2}\right]}{\cos \frac{p_{z} T}{2}} \frac{\cos \frac{k \pi T}{T_{z}}}{1-\left(\frac{2 k T}{T_{z}}\right)^{2}} .
$$

При этом номера гармоник определяют как ближайшие целые числа отношений собственных частот динамических моделей сравнивае- 
мых вариантов зубчатых передач к основной частоте:

$$
k_{i, j}=\omega_{i, j} / p_{z}
$$

При сопоставлении вариантов динамических схем в формулу (1) расчета $H_{k}$ следует дополнительно ввести коэффициент, который характеризует отношение амплитудных значений ударных импульсов, возникающих в зацеплении сопоставляемых систем - усовершен-

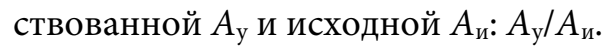

С учетом указанных дополнений формула (1) для определения динамического критерия виброактивности сопоставляемых динамических систем примет вид

$$
H_{k}=\frac{A_{\mathrm{y}}}{A_{\text {и }}} \frac{\sum_{\omega_{j} \in G} \beta_{j}\left|\tilde{q}_{m_{k}}^{(j)}\right| \omega_{j}^{2}}{\sum_{\omega_{i} \in G} \beta_{i}\left|\tilde{q}_{m_{k}}^{(i)}\right| \omega_{i}^{2}} .
$$

Оценка эффективности конструктивных решений по снижению виброактивности, основанная на анализе собственных форм колебаний, на примере зубчатых передач с составными зубчатыми колесами. В качестве ведомого зубчатого колеса используем составную конструкцию с упругим креплением и повышенной податливостью зубчатого венца [2]. Параметры зубчатой передачи: модуль $m=$ $=6,5$ мм; числа зубьев шестерни $z_{\text {ш }}=13$ и ведомого колеса $z_{\text {к }}=69$; коэффициенты смещения исходного контура шестерни $x_{\text {ш }}=0,3077$ и колеса $x_{\mathrm{K}}=0$; ширина зубчатого венца $b_{w}=$ $=0,44 \mathrm{M}$.

Расчет критерия виброактивности проведем путем сравнения виброускорений на ступице составного колеса (для зубчатой передачи с составным зубчатым колесом) и на цельном зубчатом колесе (для зубчатой передачи с цельными колесами). Динамические схемы указанных зубчатых передач приведены на рис. 2.

Параметры элементов рассматриваемых динамических схем: массы зубчатой пары с цельными колесами - шестерни $m_{ш}=5,89$ кг и колеса $m_{\mathrm{K}}=27 \mathrm{\kappa г}$; массы зубчатой пары с составным колесом - шестерни $m_{\mathrm{II}}=5,89$ кг, обода $m_{\mathrm{o}}=12$ кг и ступицы составного колеса $m_{\mathrm{c}}=$ $=15 \mathrm{\kappa r;} \mathrm{жесткости} \mathrm{-} \mathrm{зубчатого} \mathrm{сопряжения}$ $C_{3}=7,416 \cdot 10^{8} \mathrm{H} / \mathrm{M}, \quad$ вала шестерни $C_{\text {в.п1 }}=$ $=4,2 \cdot 10^{8} \mathrm{H} / \mathrm{M}$, вала колеса $C_{\text {в.п2 }}=3,35 \cdot 10^{8} \mathrm{H} / \mathrm{M}$, упругого соединения обода и ступицы составного колеса $C_{\mathrm{y} . \mathrm{c}}=1,962 \cdot 10^{8} \mathrm{H} / \mathrm{M}$; основная частота $p_{z}=244,5 \mathrm{c}^{-1}$; период зубцовой частоты
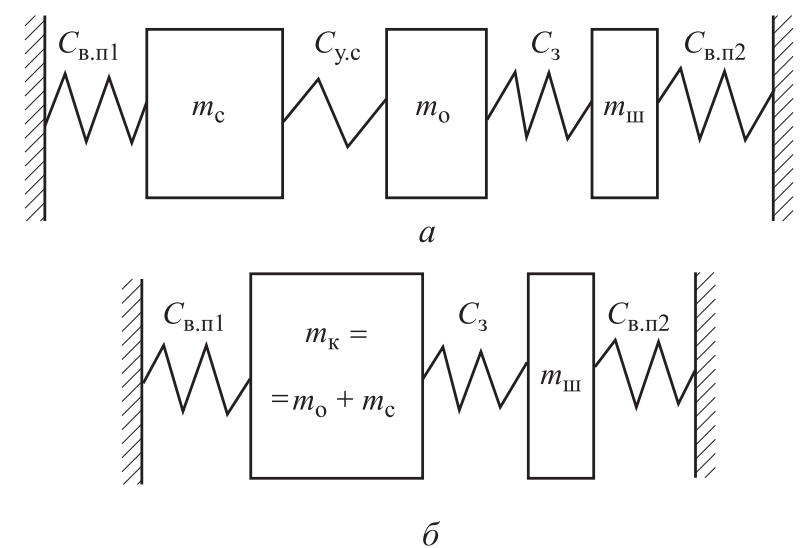

Puc. 2. Динамические схемы зубчатых передач: $a-$ с составным зубчатым колесом; 6 - с цельными колесами

$T_{z}=2,57 \cdot 10^{-2}$ с; угловая скорость ведомого колеca $\omega_{2}=3,54 \mathrm{c}^{-1}$.

Параметры ударного импульса взаимодействующих зубчатых профилей определяют из зависимостей, приведенных в работах $[8,9]$.

Амплитудное значение $F_{\max }$ находят из трансцендентного уравнения

$$
F_{\max }^{2} \ln \frac{\theta F_{\max }}{2 \pi b_{w}\left(\rho_{1}+\rho_{2}\right) e^{0,314}}=-\frac{2 \pi b_{w} m_{\text {пр }}}{\theta} v_{0}^{2},
$$

где $\theta-$ константа материала зубчатых колес, $\theta=4\left(1-\mu^{2}\right) / E \quad(\mu-$ коэффициент Пуассона, $E$ - модуль упругости); $\rho_{1}$ - радиус кривизны профиля ведущего зуба в теоретической точке начала зацепления; $\rho_{2}-$ радиус кривизны ведомого зуба в вершине; $m_{\text {пр }}$ - приведенная масса зубчатой пары; $v_{0}-$ скорость кромочного удара [6].

В выражении (6)

$$
v_{0}=\omega_{2} \sqrt{2 \delta a},
$$

где $\delta=w_{0}+\Delta t_{0}-$ приведенная ошибка зубьев $\left(w_{0}-\right.$ сближение по нормали неударяющейся пары зубьев в момент времени, соответствующей началу входа зуба в зацепление; $\Delta t_{0}-$ превышение основного шага ведомого колеса над основным шагом ведущего); $a=\rho_{2}\left(\rho_{1}+\rho_{2}\right) / \rho_{1}$.

Длительность импульса

$$
T=\frac{v_{0} m_{\text {пр }}}{F_{\max }}\left[2 S_{0}+\frac{S_{1}}{\tau}+\frac{S_{2}}{\tau^{2}}+\frac{S_{3}}{\tau^{3}}+\ldots\right],
$$

где $S_{0}=1,571 ; S_{1}=1,089 ; S_{2}=0,031 ; S_{3}=0,003$; $\tau=2 \pi b_{w} v_{0}^{2} m_{\text {пр }} /\left(\theta F_{\max }^{2}\right)-$ безразмерная величина.

Среднее значение функции податливости

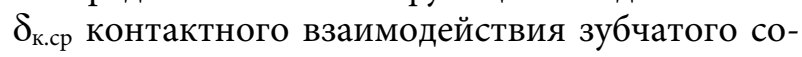




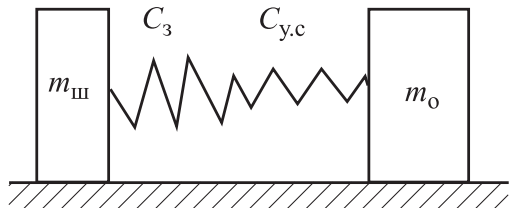

Puc. 3. Расчетная схема шестерни и деформируемого обода составного зубчатого колеса

пряжения в промежутке изменения ударной силы $0 \leq F \leq F_{\max }$ определяем по формуле [8]

$$
\delta_{\text {к.ср }}=-\frac{\theta}{2 \pi b_{w}}\left(\ln \frac{\theta e^{0,186} F_{\max }}{2 \pi b_{w}\left(\rho_{1}+\rho_{2}\right)}-1\right) .
$$

Расчетные параметры зубчатых колес: радиусы кривизны ножки зуба - шестерни $\rho_{1}=2,815 \cdot 10^{-3}$ м и колеса $\rho_{2}=93,87 \cdot 10^{-3}$ м; параметр $a=3,223 \mathrm{м}$; упругая деформация зубьев под нагрузкой $w=24 \cdot 10^{-6} \mathrm{M}$; приведенная масса зубчатой пары из цельных колес $m_{\text {пр }}^{\text {ц }}=2,418$ кг. Для рассматриваемых зубчатых колес, изготовленных по восьмой степени точности, максимальное превышение основного шага ведущего колеса над основным шагом ведомого $\left(\Delta t_{0}\right)_{\max }=$ $=60 \cdot 10^{-6} \mathrm{M}$. В соответствии $\mathrm{c}$ выражением (7) скорость кромочного удара $v_{0}=8,24 \cdot 10^{-2} \mathrm{M} / \mathrm{c}$.

В результате расчетов, проведенных для зубчатой пары с цельными колесами по выражениям (6), (8), (9), получены следующие значения искомых параметров:

$$
\begin{aligned}
& F_{\max }^{\amalg}=4418 \mathrm{H} ; \\
& T^{\Perp}=1,454 \cdot 10^{-4} \mathrm{c} ; \\
& \delta_{\text {К. } . p}^{\amalg}=86,88 \cdot 10^{-11} \mathrm{M} / \mathrm{H} .
\end{aligned}
$$

Для зубчатой пары с составным зубчатым колесом при расчете параметров ударного импульса в зубчатом сопряжении необходимо учитывать податливость обода составного зубчатого колеса [2]. В этом случае целесообразно рассматривать двухмассовую расчетную схему шестерни и обода с дополнительной жесткостью в окружном направлении упругого соединения обода со ступицей (рис. 3), присоединенной к контактной жесткости сопряженных зубьев.

В случае последовательного соединения жесткостей их податливости суммируют. Для деформируемого обода податливость $\delta_{\mathrm{y.c}}=$ $=1 / C_{\mathrm{y} . \mathrm{c}}=509,7 \cdot 10^{-11} \mathrm{~m} / \mathrm{H}$. Тогда суммарное (эквивалентное) значение податливости упругого сопряжения шестерни и обода составного колеса

$$
\begin{aligned}
\delta_{\text {экв }}=\delta_{\text {к.ср }}+\delta_{\text {у.с }}=86,88 \cdot 10^{-11}+509,7 \cdot 10^{-11}= \\
=596,58 \cdot 10^{-11} \mathrm{M} / \mathrm{H} .
\end{aligned}
$$

Параметры ударного импульса для пары шестерня - обод с большой степенью точности определяют по приближенным зависимостям [9]

$$
\begin{aligned}
& F_{\max }^{\mathrm{c}}=v_{0} \sqrt{\frac{m_{\text {пр }}^{\mathrm{c}}}{\delta_{\text {экв }}}} ; \\
& T^{\mathrm{c}}=\pi \sqrt{\delta_{\text {экв } m_{\text {пр }}^{\mathrm{c}}}} .
\end{aligned}
$$

После подстановки приведенной массы пары шестерня - обод $m_{\text {пр }}^{\mathrm{c}}=2,365$ кг в формулы (10), (11) получим следующие значения искомых параметров:

$$
\begin{aligned}
& F_{\max }^{\mathrm{c}}=8,24 \cdot 10^{-2} \sqrt{\frac{2,365}{596,58 \cdot 10^{-11}}}=1641 \mathrm{H} ; \\
& T^{\mathrm{c}}=\pi \sqrt{596,58 \cdot 10^{-11} \cdot 2,365}=3,732 \cdot 10^{-4} \mathrm{c} .
\end{aligned}
$$

В таблице приведены значения нормированных амплитуд собственных форм для цельного зубчатого колеса $\tilde{q}_{ц m_{k}}^{(i)}$ и ступицы составного колеса $\tilde{q}_{c m_{k}}^{(j)}$, собственных частот динамических моделей передач с составным колесом $\left(\omega_{j}\right)$ и цельными колесами $\left(\omega_{i}\right)$ (см. рис. 2$)$ [2], а также значения номеров гармоник $k_{i, j}$ и весовых коэффициентов $\beta_{i, j}$, вычисленных соответственно по формулам (4) и (3).

После подстановки в формулу (5) расчетных значений из таблицы и амплитудных значений ударных импульсов для зубчатых передач с составным колесом $A_{\mathrm{y}}=F_{\max }^{\mathrm{c}}$ и с цель-

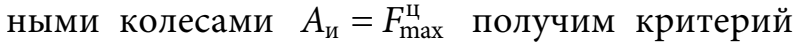
виброактивности сопоставляемых динамических схем

$$
\begin{gathered}
H_{k}=\frac{1641}{4418}\left(0,937 \cdot 0,26 \cdot 4430^{2}+0,8629 \cdot 0,473 \times\right. \\
\left.\times 6790^{2}+0,3575 \cdot 0,019 \cdot 15300^{2}\right) /(0,9880 \cdot 0,562 \times \\
\left.\times 4640^{2}+0,9019 \cdot 0,146 \cdot 14300^{2}\right)=0,250 .
\end{gathered}
$$

Значение критерия виброактивности, рассчитанное в работе [2] с помощью эмпирического поправочного коэффициента, учитывающего динамическую нагрузку в зубчатом зацеплении, составило 0,615. При выполнении вычислений априори была принята прямоугольная форма импульса в зубчатом сопряжении. 
Значения собственных частот и нормированных амплитуд собственных форм динамических моделей

\begin{tabular}{|c|c|c|c|c|c|c|c|c|c|c|}
\hline \multicolumn{9}{|c|}{ Зубчатая передача } \\
\hline$j$ & $\omega_{j}, \mathrm{c}^{-1}$ & $\tilde{q}_{\mathrm{c} m_{k}}^{(j)}$ & $k_{j}$ & $\beta_{j}$ & $i$ & $\omega_{i}, \mathrm{c}^{-1}$ & $\tilde{q}_{ц}^{(i)} m_{k}$ & $k_{i}$ & $\beta_{i}$ \\
\hline 1 & 4430 & 0,260 & дей0,9 & 0,9370 & 1 & 4640 & 0,562 & 20 & 0,9880 \\
\hline 2 & 6790 & 0,473 & 27 & 0,8629 & 2 & 14300 & 0,146 & 58 & 0,9019 \\
\hline 3 & 15300 & 0,019 & 63 & 0,3575 & - & - & - & - & - \\
\hline
\end{tabular}

Полученное с учетом уточненной формы ударного импульса значение критерия виброактивности $H_{k}=0,25$ ближе к экспериментальным результатам, приведенным в работе [10], где средние квадратические значения виброускорений у составных зубчатых колес в 2-3 раза (а на отдельных частотах - в 5-6 раз) меньше, чем у серийных цельных.

Следует отметить, что значение критерия $H_{k}$ зависит от режима работы зубчатой передачи. Это объясняется тем, что формула (3) для весовых коэффициентов $\beta_{i, j}$ содержит период зубцовой частоты $T_{z}$, определяемый по выражению (2) через частоту вращения зубчатого колеса $n$. Кроме того, в отличие от ранее рассмотрассмотренного подхода, в котором отношение времени действия импульса к периоду зубцовой частоты принято постоянным и равным $T / T_{z}=0,1$, в предлагаемой методике это отношение является расчетным. Так, для зубчатой пары из цельных колес $T^{\Perp} / T_{z}=0,565 \cdot 10^{-2}$, а для составных колес $T^{\mathrm{c}} / T_{z}=1,448 \cdot 10^{-2}$. Как видно, расчетные значения отношений существенно отличаются от принятого в прежней методике $T / T_{z}=0,1$.

\section{Выводы}

1. Отличительной особенностью предложенного метода оценки снижения уровня виброактивности зубчатых передач вследствие усовершенствования их конструкции является возможность определения относительных значений амплитуд на резонансных частотах их собственных форм колебаний без учета действия диссипативных сил. Используемая ранее оценка виброактивности, основанная на априорном принятии прямоугольной формы ударного импульса, приводит к существенно заниженным результатам.

2. Показано, что уточненное представление формы ударного импульса в виде полуволны синусоиды и его длительности на примере снижения виброактивности зубчатой передачи путем применения составного зубчатого колеса дает значение критерия виброактивности $H_{k}$ более близкое к экспериментальным результатам $(0,25$ вместо 0,615). При этом полученные при испытаниях средние квадратические значения виброускорений у составных зубчатых колес в 2-3 раза (а на отдельных частотах - в 5-6 раз) меньше, чем у серийных цельных.

\section{Литература}

[1] Гринкевич В.К., Овчинников Н.Ф. Оптимизация собственных форм динамической системы. В кн.: Методы создания машин в малошумном исполнении. Москва, Наука, 1978, c. 49-51.

[2] Берестнев О.В., Гоман А.М., Ишин Н.Н. Аналитические методы механики в динамике приводов. Минск, Навука і тэхніка, 1992. 238 с.

[3] Берестнев О.В., Гоман А.М., Скороходов А.С. Сравнительная оценка виброактивности зубчатых передач методом разложения движения по собственным формам. Доклады АНБ, технические науки, 1994, т. 38, № 3, с. 115-117.

[4] Ишин Н.Н., Леванцевич М.А., Максимченко Н.Н., Гоман А.М. Исследование виброакустических характеристик конических зубчатых передач с покрытием. Упрочняющие технологии и покрытия, 2006, № 11, с. 43-49.

[5] Лойцянский Л.Г., Лурье А.И. Курс теоретической механики. Т. 2: Динамика. Москва, Дрофа, 2006. 720 с.

[6] Диментберг Ф.М., Колесников К.С., ред. Вибрации в технике: справочник. Т. 3. Москва, Машиностроение, 1980. 544 с. 
[7] Ишин Н.Н., Гоман А.М., Скороходов А.С., Гаврилов С.А. Диагностика технического состояния приводных зубчатых механизмов на основе анализа ударных процессов в зубчатых передачах. Техническая диагностика и неразрушающий контроль, 2012, № 4, c. 43-45.

[8] Ишин Н.Н., Гоман А.М., Скороходов А.С. Исследование параметров ударного импульса в зубчатом зацеплении прямозубых цилиндрических зубчатых колес. Механика машин, механизмов и материалов, 2011, № 3, с. 19-23.

[9] Ишин Н.Н., Гоман А.М., Скороходов А.С. Исследование ударного взаимодействия прямозубых зубчатых колес применительно к задачам вибродиагностики. Определение параметров ударного импульса в зубчатом зацеплении прямозубых цилиндрических зубчатых колес. Весці НАН Белрусі, серыя фізіко-тэхніческіх навук, 2012, № 1, с. 65-71.

[10] Берестнев О.В. Самоустанавливающиеся зубчатые колеса. Минск, Навука і тэхніка, 1983. 312 c.

\section{References}

[1] Grinkevich V.K., Ovchinnikov N.F. Optimizatsiia sobstvennykh form dinamicheskoi sistemy. $V$ kn.: Metody sozdaniia mashin v maloshumnom ispolnenii [Optimization of own forms of a dynamic system. The book Methods of making cars in low-noise performance]. Moscow, Nauka publ., 1978, pp. 49-51.

[2] Berestnev, O.V., Goman A.M., Ishin N.N. Analiticheskie metody mekhaniki v dinamike privodov [Analytical methods of mechanics in the dynamics of the drives]. Minsk, Navuka i tekhnika publ., 1992. $238 \mathrm{p}$.

[3] Berestnev O.V., Goman A.M., Skorokhodov A.S. Sravnitel'naia otsenka vibroaktivnosti zubchatykh peredach metodom razlozheniia dvizheniia po sobstvennym formam [Comparative evaluation vibroaktivnosti gears by expansion movement by ownership]. Doklady ANB, tekhnicheskie nauki [Reports of the NSA, engineering science]. 1994, vol. 38, no. 3, pp. 115-117.

[4] Ishin N.N., Levantsevich M.A., Maksimchenko N.N., Goman A.M., Issledovanie vibroakusticheskikh kharakteristik konicheskikh zubchatykh peredach s pokrytiem [The research of vibroacoustic characteristics of conic tooth gearings with a coating]. Uprochniaiushchie tekhnologii i pokrytiia [Strengthening Technologies and Coatings]. 2006, no. 11, pp. 43-49.

[5] Loitsianskii L.G., Lur'e A.I. Kurs teoreticheskoi mekhaniki. T. 2: Dinamika [Course of theoretical mechanics. Vol. 2: Dynamics]. Moscow, Drofa publ., 2006. 720 p.

[6] Vibratsii v tekhnike: Spravochnik [Vibration technique: Directory]. Ed. Dimentberg F.M., Kolesnikov K.S., Moscow, Mashinostroenie publ., 1980. 544 p.

[7] Ishin N.N., Goman A.M., Skorokhodov A.S., Gavrilov S.A. Diagnostika tekhnicheskogo sostoianiia privodnykh zubchatykh mekhanizmov na osnove analiza udarnykh protsessov $\mathrm{v}$ zubchatykh peredachakh [Diagnostics of technical condition of the drive gear mechanism based on the analysis of impact processes in the gears]. Tekhnicheskaia diagnostika $i$ nerazrushaiushchii kontrol' [Technical Diagnostics and Nondestructive Testing]. 2012, no. 4, pp. 43-45.

[8] Ishin N.N., Goman A.M., Skorokhodov A.S. Issledovanie parametrov udarnogo impul'sa v zubchatom zatseplenii priamozubykh tsilindricheskikh zubchatykh koles [Investigation of the parameters of the shock pulse in the spur gears gearing]. Mekhanika mashin, mekhanizmov $i$ materialov [Mechanics of Machines, Mechanisms and Materials]. 2011, no. 3, pp. 19-23.

[9] Ishin N.N., Goman A.M., Skorokhodov A.S. Issledovanie udarnogo vzaimodeistviia priamozubykh zubchatykh koles primenitel'no $\mathrm{k}$ zadacham vibrodiagnostiki. Opredelenie parametrov udarnogo impul'sa v zubchatom zatseplenii priamozubykh tsilindricheskikh zubchatykh koles [Research of shock interaction of spur cogwheels according to vibrodiagnostic problems. Definition of parameters of a shock impulse in spur cylindrical cogwheels]. Vestsi NAN Belarusi, seryia fiziko-tekhnicheskikh navuk [Proceedings of the National Academy of Sciences of Belarus. Series of physical-technical sciences]. 2012, no. 1, pp. 65-71.

[10] Berestnev O.V. Samoustanavlivaiushchiesia zubchatye kolesa [Aligning gears]. Minsk, Navuka i tekhnika publ., 1983. 312 p. 


\section{Информация об авторах}

ИШИН Николай Николаевич (Минск) - доктор технических наук, доцент, директор научно-технического центра (НТЦ) «Карьерная техника». Государственное научное учреждение «Объединенный институт машиностроения» Национальной академии наук Беларуси (220072, Минск, Республика Беларусь, Академическая ул., д. 12, е-mail: nik_ishin@mail.ru).

ГОМАН Аркадий Михайлович (Минск) - кандидат технических наук, доцент, начальник отдела динамического анализа и вибродиагностики машин НТЦ «Карьерная техника». Государственное научное учреждение «Объединенный институт машиностроения» Национальной академии наук Беларуси (220072, Минск, Республика Беларусь, Академическая ул., д. 12, e-mail: ark.goman@gmail.com).

СКОРОХОДОВ Андрей Станиславович (Минск) - кандидат технических наук, ведущий научный сотрудник отдела динамического анализа и вибродиагностики машин НТЦ «Карьерная техника». Государственное научное учреждение «Объединенный институт машиностроения» Национальной академии наук Беларуси (220072, Минск, Республика Беларусь, Академическая ул., д. 12, e-mail: skanst@yandex.ru).

\section{Information about the authors}

ISHIN Nikolai Nikolaevich (Minsk) - Doctor of Science (Eng.), Associate Professor, Director, Scientific and Technical Center of Quarry Machinery. The State Scientific Institution Joint Institute of Mechanical Engineering of the National Academy of Sciences of Belarus (220072, Minsk, Republic of Belarus, Akademicheskaya St., Bldg 12, e-mail: nik_ishin@mail.ru).

GOMAN Arkadiy Mikhailovich (Minsk) - Candidate of Science (Eng.), Associate Professor, Head of Department of Dynamic Analysis and Vibration Diagnostics, Scientific and Technical Center of Quarry Machinery. The State Scientific Institution - Joint Institute of Mechanical Engineering of the National Academy of Sciences of Belarus (220072, Minsk, Republic of Belarus, Akademicheskaya St., Bldg. 12, e-mail: ark.goman@gmail.com).

SKOROKHODOV Andrey Stanislavovich (Minsk) - Candidate of Science (Eng.), Leading Research Scientist, Department of Dynamic Analysis and Vibration Diagnostics, Scientific and Technical Center of Quarry Machinery. The State Scientific Institution - Joint Institute of Mechanical Engineering of the National Academy of Sciences of Belarus (220072, Minsk, Republic of Belarus, Akademicheskaya St., Bldg. 12, e-mail: skanst@yandex.ru).

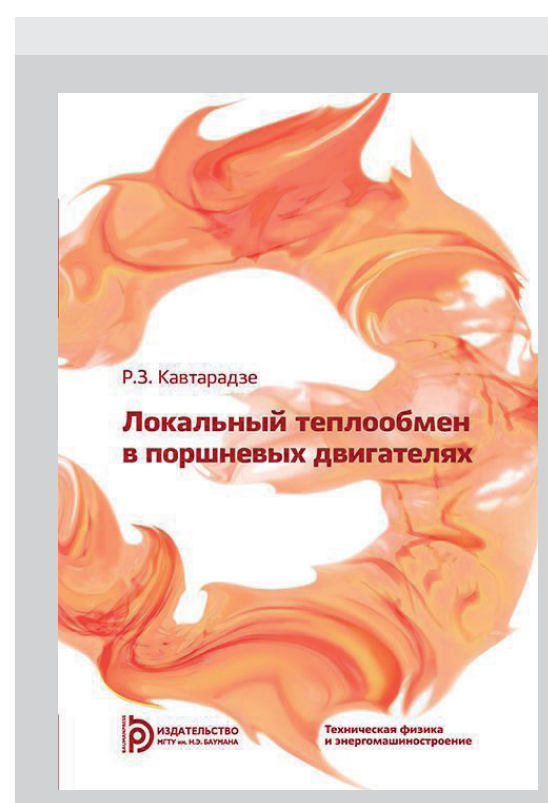

\section{В Издательстве МГТУ им. Н.Э. Баумана вышел в свет учебник P.3. Кавтарадзе «Локальный теплообмен в поршневых двигателях"}

Учебник посвящен исследованию локального теплообмена в поршневых двигателях. Значительная его часть написана на основе результатов, полученных в МГТУ им. Н.Э. Баумана. Ряд вопросов в теории поршневых двигателей рассматривается впервые. В данный учебник, написанный на основе учебного пособия с тем же названием (1-е изд. - 2001 г., 2-е изд. - 2007 г.), включены новые материалы, отражающие достижения последних лет в этой области науки. Содержание учебника соответствует курсу лекций, который автор читает в МГТУ им. Н.Э. Баумана.

Для магистрантов, аспирантов, научных и инженерно-технических работников, занимающихся созданием перспективных двигателей, а также исследованием и доводкой существующих моделей.

По вопросам приобретения обращайтесь:

105005, Москва, 2-я Бауманская ул., д. 5, стр. 1.

Тел.: +7 499 263-60-45, факс: +7 499 261-45-97;

press@bmstu.ru; www.baumanpress.ru 